\title{
Applications of MEMS-MOSFET Hybrid Switches to Power Management Circuits for Energy Harvesting Systems
}

\author{
Sang-Hun Song ${ }^{*}$, Sungmuk Kang ${ }^{*}$, Kyungjin Park ${ }^{*}$, Seunghwan Shin ${ }^{*}$, and Hoseong Kim ${ }^{\dagger}$ \\ $\grave{\dagger}^{*}$ School of Electrical and Electronics Engineering, Chung-Ang University, Seoul, Korea
}

\begin{abstract}
A hybrid switch that uses a microelectromechanical system (MEMS) switch as a gate driver of a MOSFET is applied to an energy harvesting system. The power management circuit adopting the hybrid switch provides ultralow leakage, self-referencing, and high current handling capability. Measurements show that solar energy harvester circuit utilizing the MEMS-MOSFET hybrid switch accumulates energy and charges a battery or drive a resistive load without any constant power supply and reference voltage. The leakage current during energy accumulation is less than $10 \mathrm{pA}$. The power management circuit adopting the proposed hybrid switch is believed to be an ideal solution to self-powered wireless sensor nodes in smart grid systems.
\end{abstract}

Key words: MEMS-MOSFET hybrid switch, Energy harvesting, Power management circuit, Ultralow leakage, Self-referencing, Wireless sensor nodes, Smart grid

\section{INTRODUCTION}

Batteries are widely used to power wireless sensor nodes and mobile devices, but they require periodic exchange or recharge. Instead of batteries or as an energy source for recharge, the harvested energy from light, heat, or vibration available in our environments can be utilized for low average power electronic devices that have a very low $(<1 \%)$ operation duty cycle [1], [2]. Power monitoring sensors in a smart grid system require power source, but it is impractical to intervene the high voltage power line to draw low power necessary to drive each low voltage sensors. Recently, researchers in power electronics pay attentions to power management circuits for energy harvesting due to the particular characteristics of environmental energy [3]-[7]. However, most of these reports can be applied when the harvested power is high enough to drive the system. In realistic environments, the harvested power is usually on the order of microwatt that is less than or equal to the same order of sleep mode power, and it varies significantly over time and is sporadic in some cases [8]. Therefore, the harvested power should be accumulated in a storage capacitor over time and the

Manuscript received Jun. 25, 2012; revised Sep. 28, 2012

Recommended for publication by Associate Editor Hyung-Min Ryu.

†Corresponding Author: hkim@cau.ac.kr

Tel: +82-2-820-5292, Fax: +82-2-825-9210, Chung-Ang University

* School of Electrical and Electronics Engineering, Chung-Ang University, Korea storage capacitor should be disconnected from the load during the energy accumulation stage to prevent energy leakage to the load. The storage capacitor is supposed to be connected to the load only if the accumulated energy is large enough to drive it. This means that the accumulated energy should be monitored all the time. Furthermore, in case of a wireless sensor node, after it completes sensing, data processing, and RF data transmitting, the storage capacitor should be disconnected again from the sensor node for the next energy accumulation stage. The serious problem in this autonomous scenario is that all of these functionalities require a certain amount of energy, but the only available energy is the precious harvested energy. Some of these functionalities without any stable voltage source have been partially realized by the MIT Media Laboratory group using a bipolar junction transistor (BJT), a zener diode, a MOSFET, and the low-battery-out function of a voltage regulator for a shoe-mounted piezoelectric harvesting system [9]. In this case, however, the energy loss due to the leakage currents flowing through the BJT and zener diode is inevitable during the energy accumulation stage. The electrostatically actuated microelectromechanical system (MEMS) switch might be a good candidate to provide these functionalities without any constant voltage source. Yet, it suffers from a low current handling capability and a short lifetime at the elevated currents [10]. The MEMS-switch-based power management architecture has been reported recently, but it requires reference 
voltage of $-20 \mathrm{~V}$ [11]

In this paper, we introduce the operation of the electrostatically actuated MEMS switch that has a low actuation voltage, and an autonomous operation of the harvesting circuit utilizing the MEMS-MOSFET hybrid switch for capacitive and resistive loads, without any stable power source. In the MEMS-MOSFET hybrid structure, the MEMS switch is used as a gate driver of a MOSFET. The typical example of a capacitive load is a rechargeable battery used in wireless devices such as wireless sensor nodes and mobile electronics. The result for a resistive load has been published in our previous paper [12], but it is included here again to introduce more detailed analysis and to cover up all the possible applications of the hybrid switch to the energy harvesting system. Using a fabricated MEMS switch, the MEMS-MOSFET hybrid switch is constructed and two major application circuits of energy harvesting, which are a battery charging circuit and a load driving circuit, are built and experimental results are presented.

\section{MEMS-MOSFET HYBRID SWITCH}

Fig. 1 shows a schematic diagram of a MEMS-MOSFET hybrid switch in which the MEMS switch works as a gate driver of the MOSFET switch. The MEMS switch consists of a single crystal silicon moving part and a glass substrate. The basic structure and fabrication process of MEMS switch is similar to those reported, and the MEMS switch was used as an RF signal switch [13], but in this work, the process is modified to reduce further the actuation voltage.

In the MEMS switch shown in Fig. 1, A and B are the signal lines which are separated physically with a $2.5-\mu \mathrm{m}$ gap, and A is connected to the moving part. As a contact area, gold is deposited on the bottom of the moving part. The moving part is suspended by an elastic force of the springs, and $\mathrm{C}$ is connected to an electrical ground.

When the voltage between the moving part and $\mathrm{C}$ is increased to a certain voltage, the electrostatic force becomes larger than the elastic force of the springs; hence, the moving

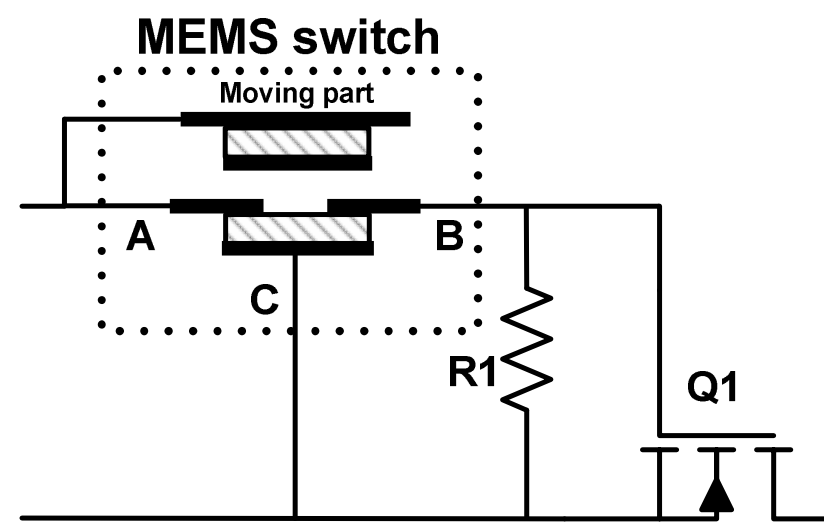

Fig. 1. Schematic diagram of the MEMS-MOSFET hybrid switch. $\mathrm{A}$ and $\mathrm{B}$ are signal lines. part goes down and the contact area connects A and B. This voltage is called the pull-in voltage of the MEMS switch. It enables the electrical signal to be delivered from A to B. When the voltage decreases under a certain level, the electrostatic force becomes smaller than the elastic force of the springs. Consequently, the moving part goes up, which disconnects A from B. This voltage is called the release or pull-out voltage of the MEMS switch. Since the signal lines are fabricated on a glass substrate and physically separated, the leakage current is almost zero during the off-state of the MEMS switch. Furthermore, since the pull-in voltage is always higher than the release voltage, the MEMS switch has a hysteretic property.

As shown in Fig. 1, the moving part is at the same potential with $\mathrm{A}$ and the MEMS switch works as a gate driver of the MOSFET. Therefore, as long as the voltage between A and $\mathrm{C}$ is lower than the pull-in voltage, the gate-source voltage is 0 $\mathrm{V}$ and keeps the MOSFET in the off-state. When the voltage between $\mathrm{A}$ and $\mathrm{C}$ increases to the pull-in voltage, $\mathrm{A}$ is connected to $\mathrm{B}$ and the gate-source voltage increases abruptly and the MOSFET switch turns on. When the voltage decreases under the release voltage, A is isolated from B and the gate-source voltage is discharged through R1 (a few M $\Omega$ ) to $0 \mathrm{~V}$ and the MOSFET switch turns off. Due to the hysteresis of the MEMS switch, the MEMS-MOSFET hybrid switch does not require the pulse generating circuit to control the MOSFET switch.

Fig. 2 shows a photograph of a (a) fabricated and (b) mounted MEMS switch. Dimensions of the designed MEMS switch are as follows: thickness of the spring $=15 \mu \mathrm{m}$, width of the spring $=4 \mu \mathrm{m}$, thickness of the membrane $=70 \mu \mathrm{m}$, gap between the electrode and switch $=2.5 \mu \mathrm{m}$, and size of

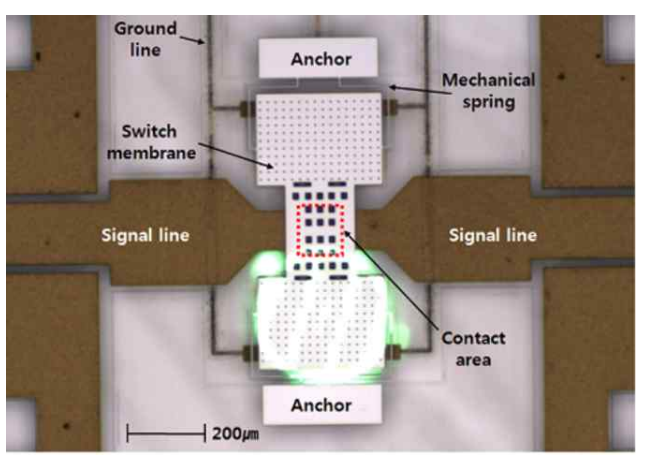

(a)

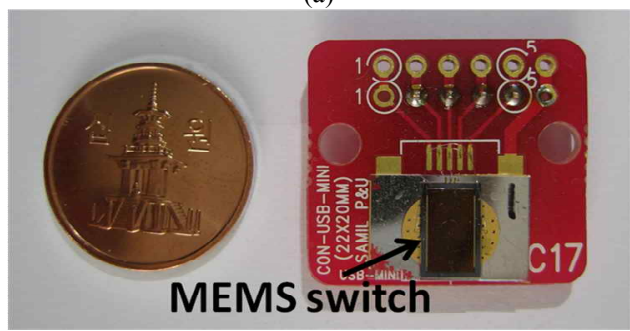

(b)

Fig. 2. Photograph of a (a) fabricated [12] and (b) mounted MEMS switch. 


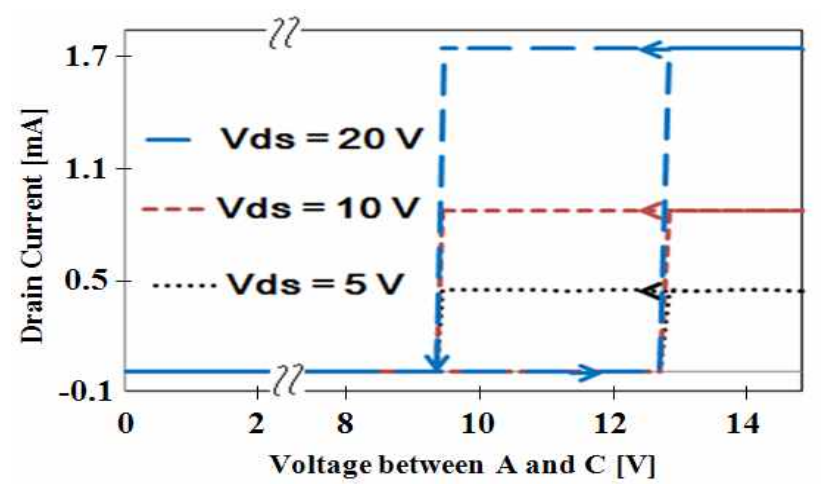

Fig. 3. Measured hysteresis characteristics of a fabricated MEMS-MOSFET switch.

the bottom electrode $=300 \mu \mathrm{m} \times 400 \mu \mathrm{m}$. Measured pull-in and pull-out voltages of the fabricated MEMS switch were 10 $\sim 13 \mathrm{~V}$ and $8 \sim 9.5 \mathrm{~V}$, respectively. These values are almost half of the values reported in [13].

The measured hysteretic property of a MEMS-MOSFET hybrid switch is shown in Fig. 3. In practice, the transition width would be zero volts, but the figure shows finite transition width due to the resolution limit of the dc power supply. The pull-in voltage and the release voltage of the MEMS-MOSFET hybrid switch are measured to be $12 \mathrm{~V}$ and $9.2 \mathrm{~V}$, respectively. Since they are determined by the mechanical structure and properties of the MEMS switch, in contrast to Schmitt trigger or comparator, the hybrid switch does not require the use of stable dc power supply and reference voltage. Therefore, employing the hybrid switch can greatly simplify a circuit and save energy.

\section{Circuit Operation AND Results}

For the first demonstration, to simulate a rechargeable battery, a $470 \mu \mathrm{F}$ capacitor is used as a capacitive load and a diode is included to prevent reverse leakage as shown in Fig. 4. As an energy harvester, we use four solar cells (with a total size of $55 \mathrm{~mm} \times 27 \mathrm{~mm}$ ) taken from commercial calculators. They are connected in series, generating an open-circuit voltage of $15 \mathrm{~V}$ on an office desk under indoor lighting conditions (300 lux).

Since the output power from the solar cells in this condition is very low, it should be firstly accumulated over

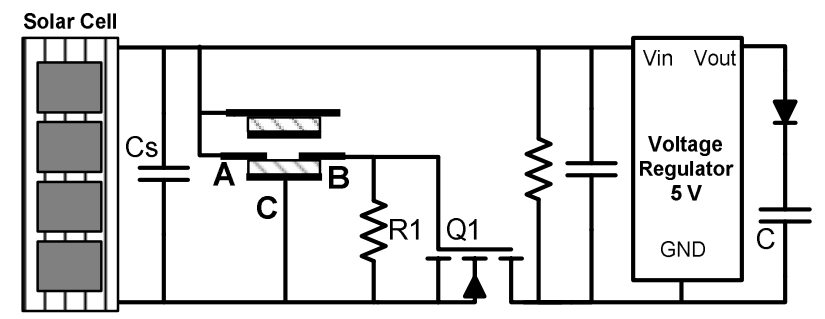

Fig. 4. A capacitor (rechargeable battery) charging circuit employing MEMS-MOSFET switch. time in a storage capacitor CS $(100 \mu \mathrm{F})$ until the accumulated energy is sufficiently high to drive the voltage regulator and the storage capacitor CS should be isolated from the following circuitry during this accumulation stage. Since the MEMS switch is normally off and gate of the MOSFET is grounded through R1 (5 M 2 ), deactivated MOSFET Q1 makes the ground of the regulator floating (as shown in Fig. 3 ) and the stored energy is not discharged to the following part of the circuit. The resistor $(750 \mathrm{k} \Omega)$ and capacitor $(100$ $\mathrm{nF}$ ) on the input side of voltage regulator are used for impedance matching [9]. As long as the solar cells convert the light power to electric power, the capacitor voltage (upper trace) gradually increases toward the solar cell's open circuit voltage as shown in Fig. 5. This voltage is also applied to the MEMS switch so that the silicon membrane starts to deflect downward due to an electrostatic force between silicon membrane and electrodes, but it does not touch the disconnected signal lines. When the storage capacitor voltage reaches the pull-in voltage $(12 \mathrm{~V})$ of the MEMS switch, the switch membrane suddenly collapses down and the gold contact on the bottom of silicon membrane closes the signal lines underneath. The voltage of $\mathrm{C}_{\mathrm{S}}$, at this moment, is applied to the gate of the MOSFET and the MOSFET is turned on. Afterward, the grounds of the storage capacitor and the voltage regulator are connected through the MOSFET, and the stored energy is delivered to the voltage regulator to charge the load capacitor. The accumulated energy in the storage capacitor is transferred to the load capacitor, resulting in rapid decrease of storage capacitor voltage (upper trace) as shown in Fig. 5. It takes $15 \mathrm{~ms}$ (not resolved in this figure due to the long time scale) for the storage capacitor voltage to deceases from $12 \mathrm{~V}$ to $9.2 \mathrm{~V}$ (the pull-out voltage), but most of this time is the turn-on delay time of the voltage regulator ( $\sim 14 \mathrm{~ms})$.

When the storage capacitor voltage is reduced to the pull-out voltage of the MEMS switch, the switch membrane returns to the up-state position and opens the signal lines. The charges on the gate are discharged through R1 to ground and the MOSFET is turned off. Then the ground reference to the voltage regulator ceases, which makes the storage capacitor open-circuited and the next energy accumulation process resumes. Fig. 5 shows that this cycle autonomously repeats until the load capacitor voltage (lower trace) increases stepwise to reach the final full-charge voltage and then the charging process ceases.

The final full-charge voltage $(4.4 \mathrm{~V})$ is determined by the regulator voltage $(5 \mathrm{~V})$ and forward voltage drop (about 0.6 $\mathrm{V})$ of the diode. Whenever the voltage of the load capacitor (or rechargeable battery) decreases for some reason, the cycle repeats except when the storage capacitor is charged again starting from the last voltage of it. The advantage of this circuit is that the energy accumulating and charging is possible under the very low illumination. During the accumulation stage, since the signal lines fabricated on glass substrate are physically separated, the leakage through the glass substrate is less than $1 \mathrm{pA}$ and out of measurement 


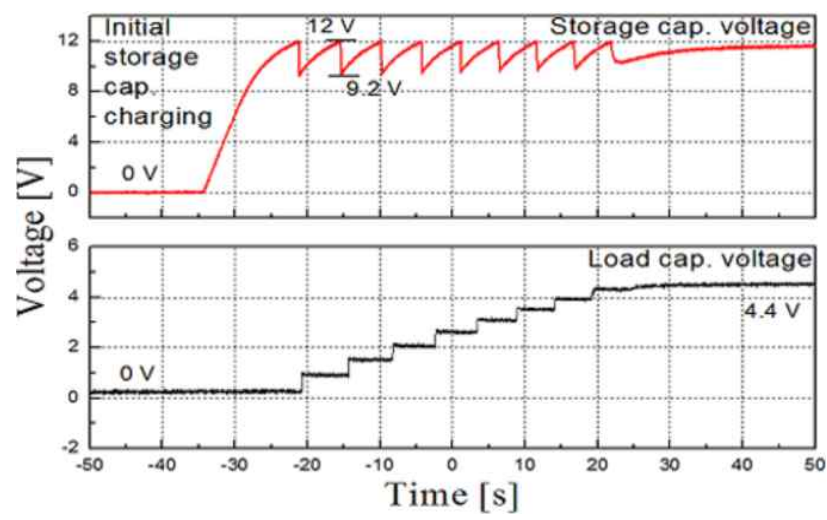

Fig. 5. Photograph Storage capacitor voltage (upper trace) and load capacitor voltage (lower trace).

range of picoammeter. In addition, since the gate of MOSFET is grounded through R1 during the accumulation stage, the drain-source leakage current is negligible. Measurements show that the leakage current is less than 10 $\mathrm{pA}$, which means it is almost completely isolated.

If the solar cells are directly connected to the voltage regulator without the MEMS-MOSFET hybrid switch under very low lighting conditions, all of the output power generated by the solar cells is consumed to supply the quiescent power of the regulator without any output; therefore, the load capacitor (battery) can never be charged. Also, when the semiconductor automatic connection circuit [9] is used, a few tens of nA leakage through BJT and zener diode develops a few $\mathrm{mV}$ on the gate. In spite of the fact that this gate voltage is lower than the threshold voltage of the MOSFET, the sub-threshold drain-source leakage current flows. This sub-threshold current exponentially increases as the gate voltage increases, which also results in the failure of energy harvesting. Moreover, when the MEMS switch is used alone as a main power line switch without the MOSFET, the MEMS switch fails after a few cycles due to the initial high peak charging current.

During the on-time of the MEMS switch, when the $100 \mu \mathrm{F}$ storage capacitor voltage decreases from $12 \mathrm{~V}$ to $9.2 \mathrm{~V}$ in 15 $\mathrm{ms}$, it discharges $3 \mathrm{~mJ}$ while $\mathrm{R} 1$ consumes only $0.3 \mu \mathrm{J}$ $(0.01 \%)$. Therefore, the energy harvesting circuit with our MEMS-MOSFET hybrid switch makes it possible to harvest energy and recharge the battery even if the generated power is extremely low in solar, piezoelectric, or thermoelectric energy harvesting systems.

The second demonstration with a resistive load, which was published in our previous paper [12], is presented here again to introduce more detailed analysis and to cover up all the possible applications of the hybrid switch to the energy harvesting system. In this demonstration, a series-connected current limiting resistor and an LED are used as a resistive load as shown in Fig. 6. The LED and resistor draw a current of $20 \mathrm{~mA}$ at $5 \mathrm{~V}$. In a practical application, this resistive load can be a sensor circuitry that includes sensors, a microprocessor, and an RF transmitter. As long as the solar cells convert the light power to electric power, the capacitor

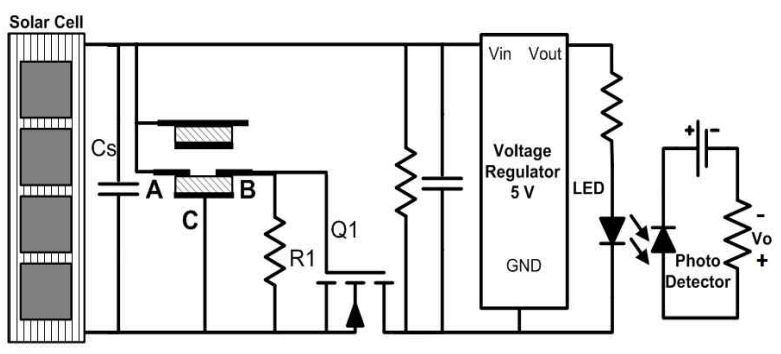

Fig. 6. A resistive load driving circuit employing MEMSMOSFET switch and measurement circuit.

voltage increases to the solar cell's open circuit voltage. As soon as the storage capacitor voltage reaches the pull-in voltage $(12 \mathrm{~V})$, the MEMS switch and MOSFET are turned on as in the first demonstration. Since the voltage regulator supplies constant voltage to the resistive load in this case, the storage capacitor voltage decreases linearly due to the constant power dissipation of the resistive load. The MEMS switch, however, does not return to its original position immediately because the electrostatic force is still larger than the restoring force of the mechanical springs. As the storage capacitor reduces closer to the pull-out voltage of the MEMS switch, the MEMS switch turns off, followed by the deactivation of MOSFET as in the first case.

Fig. 7 shows the storage capacitor voltage (upper trace) and the LED output (lower trace) measured with a photodetector as shown in Fig. 6. This LED/photodetector measurement configuration is used as a photocoupler in order to keep ground isolation during the synchronous measurement of two waveforms as shown in Fig. 7. Note that the whole cycle of accumulation, energy delivery and next energy accumulation processes are autonomously repeated as long as the light is incident to the solar cells. The higher the incident light power is, the higher the repetition rate is. To accomplish this operation without our MEMS-MOSFET hybrid switch, a comparator [14] or Schmitt trigger circuit can be used; however, these components require another external stable power source for the operation and reference voltage, which consumes energy all the time. In this demonstration, the discharging time is about $300 \mathrm{~ms}$ and predominantly determined by the impedance of the resistive load rather than the turn-on delay time of the voltage regulator. During the discharge of the $100 \mu \mathrm{F}$ storage capacitor from $12 \mathrm{~V}$ to $9.2 \mathrm{~V}$ in $300 \mathrm{~ms}$, it discharges energy of $3 \mathrm{~mJ}$ and while R1 consumes $6 \mu \mathrm{J}$ since on-time of the hybrid switch is 20 times longer than that of the first demonstration. Therefore, when the load is capacitive, a regulator with short turn-on delay time should be used for the efficient charging. When the load is resistive, the operation time should be minimized for the efficient operation, which means that high frequency data transmission is more beneficial in wireless sensor nodes if the power consumption $\mathrm{RF}$ transmitter is constant over the carrier frequency.

Using the results mentioned above, the hybrid switch seems to be applied to reduce the standby power. Absolute 


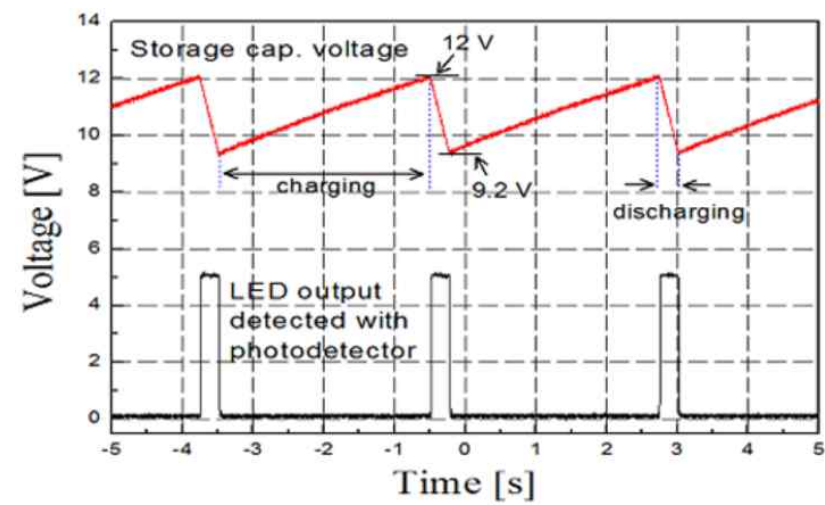

Fig. 7. Storage capacitor voltage (upper trace) and LED output measured with a photodetector (lower trace).

zero standby power has been achieved using laser light power transmission in [15]. Since semiconductor switches were used to control the power relay that switches $220 \mathrm{~V}$ power line in that report, it took several seconds to turn on/off the appliance due to the losses in semiconductor switch. We are working to replace the semiconductor switches with hybrid switch and it is believed that the turn on/off time and required laser power would be further reduced.

The first advantage of the MEMS-MOSFET hybrid switch is near-zero power loss in the energy accumulation process. Since the signal lines of the MEMS switch are fabricated on the glass substrate and physically disconnected, the leakage through the glass substrate is negligible (less than $12 \mathrm{pA}$ ). When semiconductor devices are used as in the MIT circuit, the inevitable leakage current (order of $1 \mathrm{nA}$ ) through a BJT and a zener diode prolongs the storage time. To make the matter worse, charge buildup on the gate of MOSFET and following gate voltage increase due to this leakage current force the MOSFET operate in the subthreshold or triode region, which could result in the failure of ground floating and, consequently, the failure of energy accumulation. The second advantage is its simplicity and ultralow operation power. The pulse forming circuit composed of a few devices is replaced with a single MEMS switch for gate driving and, more importantly, MEMS switch requires neither any external power supply nor any reference voltage that consume power all the time. Finally, since the main power is delivered to the load via the MOSFET and not the MEMS switch, a sufficient amount of current can be handled with this hybrid switch. We believe that these advantages make the MEMS-MOSFET hybrid switch a solution component in developing the practical energy harvesting power circuit for the self-sustaining wireless ubiquitous sensor network (WUSN), where sensor nodes are in standby mode for almost $99 \%$ of the time and harvested energy is limited. Power dissipation in the gate resistor R1 during on-state of the MEMS switch might be problematic, but the operation duty cycle is so small in the practical WUSN that the dissipation in the resistor has little effect on the performance.

\section{ACKNOWLEDGMENT}

This research was supported by the Chung-Ang University Research Scholarship Grant in 2010.

\section{REFERENCES}

[1] R. Amirtharajah and A. P. Chandrakasan, "Self-powered signal processing using vibration-based power generation," IEEE J. Solid-State Circuits, Vol. 33, No. 5, pp. 687-695, May 1998.

[2] C. O. Mathúna, T. O'Donnell, R. V. Martinez-Catala, J. Rohan, and B. O'Flynn, "Energy harvesting for long-term deployable wireless sensor networks," Talanta, Vol. 75, No. 3, pp. 613-623, May 2008.

[3] N. Kong and D. Ha, "Low-power design of a self-powered piezoelectric energy harvesting system with maximum power point tracking," IEEE Trans. Power Electron., Vol.27, No. 5, pp.2298-2308, May 2012.

[4] G. D. Szarka, B. H. Stark, and S. G. Burrow, "Review of power conditioning for kinetic energy harvesting system," IEEE Trans. Power Electron., Vol.27, No. 2, pp.803-815, Feb. 2012

[5] S. Cheng, R. Sathe, R. Natarajan, and D. P. Arnold, "A voltage-multiplying self-powered $\mathrm{AC} / \mathrm{DC}$ converter with $0.35-\mathrm{V}$ minimum input voltage for energy harvesting applications," IEEE Trans. Power Electron., Vol.26, No. 9, pp.2542-2549, Sep. 2011.

[6] Y. Sun, N. H. Hieu, C-J Jeong, S-G Lee, “An Integrated High-Performance Active Rectifier for Piezoelectric Vibration Energy Harvesting System," IEEE Trans. Power Electron., Vol.27, No. 2, pp.623-627, Feb. 2012.

[7] G.-B. Chung and K. D. T. Ng, "Analysis of an AC/DC Resonant Pulse Power Converter for Energy Harvesting Using a Micro Piezoelectric Device," Journal of Power Electronics, Vol. 5, No.4, pp.247-256, Oct. 2005.

[8] S. Roundy, P.K. Wright, and J. M. Rabaey, Energy Harvesting for Wireless Sensor Networks, Kluwer Academic Publishers, Chap. 1, 2003.

[9] N. S. Shenck and J. A. Paradiso, "Energy harvesting with shoe-mounted piezoelectrics," IEEE Micro, Vol. 21, No. 3, pp. 30-42, May/Jun. 2001.

[10] R. Kasim, B. Kim, and J. Drobnik, "Advanced MEMS for high power integrated distribution systems," Proc. 2005 ICMENS, p. 247, Jul. 2005.

[11] T. Shimamura, M. Ugajin, K. Kuwabara, K. Takagahara, K. Suzuki, H. Morimura, M. Harda, and S. Mutoh, "MEMS-switch-based Power Management with Zeropower Voltage Monitoring for Energy Accumulation Architecture on Dust-size Wireless Sensor Nodes," IEEE Symposium on VLSI Circuits, Digest of technical papers, pp. 276-277, Jun. 2011.

[12] H. Kim, S. M. Kang, K. J. Park, Y.-R. Kim, and C.-W. Baek, "Micro-electro-mechanical system/ field- effecttransistor hybrid switch for energy harvesting system," Jpn. J. Appl. Phys., Vol. 49, No. 6, pp. 06GN19-1 06G N19-3, Jun. 2010.

[13] J.-M. Kim, J.-H. Park, C.-W. Baek, and Y.-K. Kim, "The SiOG-based Single-crystalline Silicon (SCS) RF MEMS Switch with Uniform Characteristics," J. Micro-electro -mech. Syst., Vol. 13, No. 6, pp. 1036-1042, Dec. 2004.

[14] S.C.L. Yuen, J.M.H. Lee, W.J. Li, and P.H.W. Leong, “An AA-sized vibration-based microgenerator for wireless sensors," IEEE Pervasive Comput., Vol. 6, No. 1, pp. 
64-72, Jan. 2007.

[15] S. M. Kang, K. J Park, S. H. Shin, K. S. Chang, and H. Kim, "Zero standby power remote control system using light power transmission," IEEE Trans. Consum. Electron., Vol. 57, No. 4, pp.1622-1627, Nov. 2011.

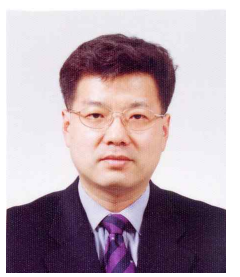

Sang-Hun Song was born in Gwangju, Korea, in 1963. He received the B.S. degree in Electronics Engineering from Seoul National University, Seoul, Korea, in 1986. $\mathrm{He}$ received the M.S. and Ph.D. degrees in Electrical Engineering from Princeton University, NJ, U.S.A., in 1988 and 1997, respectively. In 1997, he worked at LG Semicon. Ltd. as a Principal Engineer. In 1999, he worked as a Research Fellow at iQUIPS, University of Seoul, Seoul, Korea. In 2000, he worked as a Research Assistant Professor at the Electronics Engineering Department, Korea University, Seoul, Korea. Since 2001, he has been with the School of Electrical and Electronics Engineering, Chung-Ang University, Seoul, Korea, where he is currently a Professor. His research interests include electronic devices and circuits, and optoelectronics.

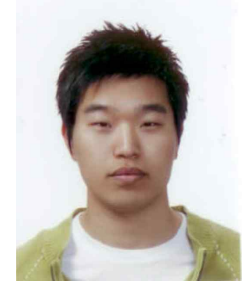

Sungmuk Kang was born in Pohang, Korea, in 1984. He received the B.S. and M.S. degrees in electrical and electronics engineering from Chung-Ang University, Seoul, Korea, in 2007 and 2009, respectively. Currently, he is working toward the Ph.D. degree in electrical and electronics engineering at Chung-Ang University. His research interests include energy-harvesting circuit design, wireless sensor networks, power management systems, and Laser/LED applications.

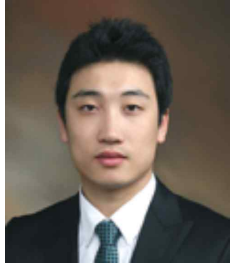

Kyungjin Park was born in Busan, Korea, in 1981. He received the B.S. and M.S. degrees in electrical and electronics engineering from Chung-Ang University, Seoul, Korea, in 2008 and 2010, respectively. He is currently working toward the Ph.D. degree in electrical and electronics engineering at Chung-Ang University. His research interests include MEMS, energy harvesting, wireless sensor networks, power management systems, and laser and LED applications.

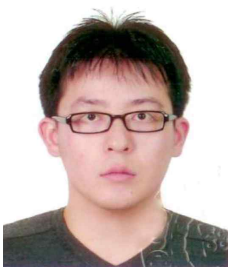

Seunghwan Shin was born in Busan, Korea in 1983. He received the B.S. and M.S. degrees in electrical and electronics engineering from Chung-Ang University, Seoul, Korea, in 2010 and 2012, respectively. He is currently working with Halla Climate Control Corporation. His research interests include energy harvesting, wireless sensor networks, and power management systems.

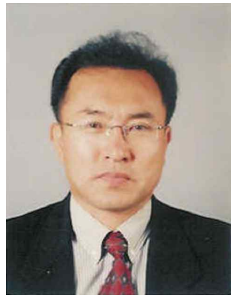

Hoseong Kim was born in Seoul, Korea, in 1957. He received the B.S. degrees in electrical engineering from Seoul National University, Seoul, Korea, in 1980. He received the M.S. and Ph.D. degrees in electrical and computer engineering from State University of New York, Buffalo, in 1988 and 1992, respectively. He joined Chung-Ang University in 1993 and is currently a Professor in the School of Electrical and Electronics Engineering. He is currently the head of the Circuit Design and Light Applications Laboratory. His current research interests include energy harvesting, wireless sensor networks, optical sensors and metrology, and circuit design. 